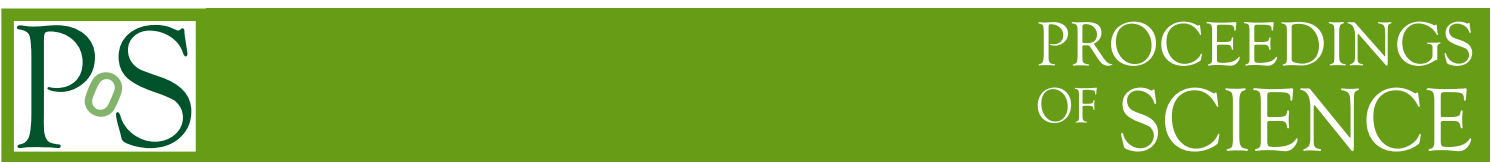

\title{
CPT violation and neutrino oscillation experiments
}

\section{Christoph Andreas Ternes ${ }^{a, *}$}

a INFN, Sezione di Torino, Via P. Giuria 1, I-10125 Torino, Italy

E-mail: ternes@to.infn.it

In this talk I discuss neutrino oscillations with different oscillation parameters for neutrinos and antineutrinos. According to the CPT theorem neutrinos and antineutrinos should behave the same, except for effects of the CP-phase $\delta$. I discuss the bounds that can be put on the differences between neutrino and antineutrino parameters from current neutrino and antineutrino data. I also discuss prospects for future experiments, and in particular some interesting scenarios that arise in the context of CPT violation, such as impostor solutions. I also discuss mimicking effects between CPT-violating neutrino oscillations and non standard neutrino interactions.

7th Symposium on Prospects in the Physics of Discrete Symmetries (DISCRETE 2020-2021)

29th November - 3rd December 2021

Bergen, Norway

${ }^{*}$ Speaker 


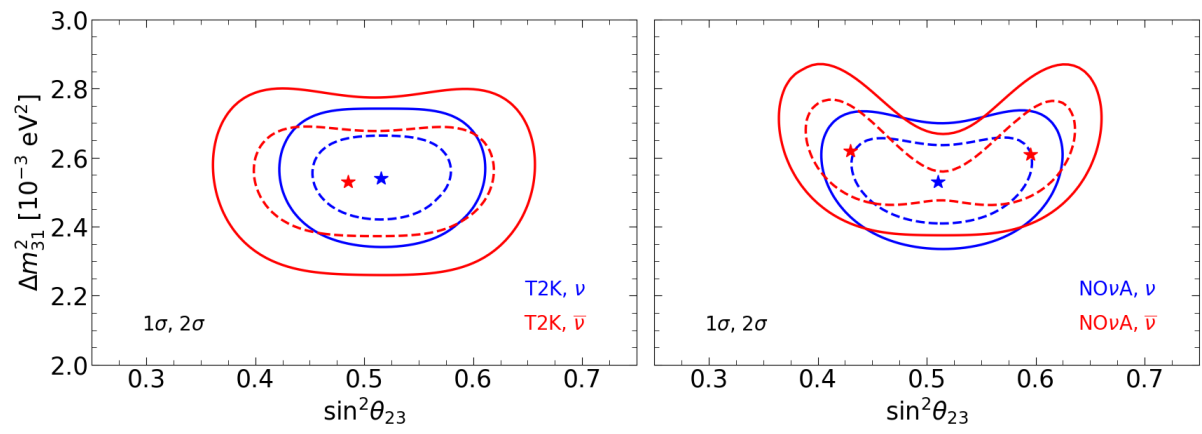

Figure 1: $1 \sigma$ (dashed) and $2 \sigma$ (solid) allowed regions in the $\sin ^{2} \theta_{23}-\Delta m_{31}^{2}$ plane $\left(\sin ^{2} \bar{\theta}_{23}-\Delta \bar{m}_{31}^{2}\right.$ plane for antineutrinos) for $\mathrm{T} 2 \mathrm{~K}$ (left) and $\mathrm{NO} v \mathrm{~A}$ (right) neutrino (blue) and antineutrino (red) data. The stars correspond to the best fit values obtained in each analysis.

\section{Introduction}

CPT invariance is one of the most important predictions of local, relativistic quantum field theory. One of the predictions of CPT invariance is that particles and antiparticles have the same masses and, if unstable, the same lifetimes. Proving the CPT theorem requires only three ingredients: Lorentz invariance, Hermiticity of the Hamiltonian, Locality. Therefore, a violation would result in a huge impact on particle physics. Here we will see how well neutrinos can test the predictions of the CPT theorem by comparing neutrino and antineutrino oscillations. A different pattern would be a possible indication of CPT violation. We will first discuss the limits on differences between neutrino and antineutrino oscillation parameters as obtained from current data. Next, we discuss how much these bounds can be improved with the next generation experiment DUNE. In this context we discuss some interesting features, like imposter solutions and mimicking effects between CPT violation and neutrino non-standard interactions (NSI).

\section{Current bounds on CPT violation}

To compute current bounds, we analyze the data from neutrino and antineutrino oscillation experiments separately. In particular, we use data from the accelerator experiments T2K [1] and $\mathrm{NO} v \mathrm{~A}$ [2] and the latest antineutrino data from the reactor experiments Daya Bay [3] and RENO [4].

We perform a fit to neutrino and to antineutrino data separately and calculate $\chi^{2}(\Delta x)=$ $\chi^{2}(|x-\bar{x}|)=\chi^{2}(x)+\chi^{2}(\bar{x})$, where $x$ refers to any of the oscillation parameters and $\bar{x}$ to its antineutrino counterpart. Since the effect of solar neutrino oscillation parameters is not appreciable in the experiments discussed in this paper, we keep them fixed throughout our analysis at $\sin ^{2} \theta_{12}=$ $\sin ^{2} \bar{\theta}_{12}=0.32$ and $\Delta m_{21}^{2}=\Delta \bar{m}_{21}^{2}=7.5 \times 10^{-5} \mathrm{eV}^{2}$ [5]. Our results for the analysis of T2K (left) and $\mathrm{NO} v \mathrm{~A}$ (right) neutrino (blue) and antineutrino (red) data in the atmospheric plane is shown in Fig. 1. Note that we always fit all the parameters at the same time. In all the figures the parameters not plotted have been marginalized over. In the left panel, we observe a very good agreement in the regions preferred by $\mathrm{T} 2 \mathrm{~K}$ neutrino and antineutrino data. As expected, the sensitivity is much better in the neutrino channel, but still one can appreciate a total overlap between the two regions 

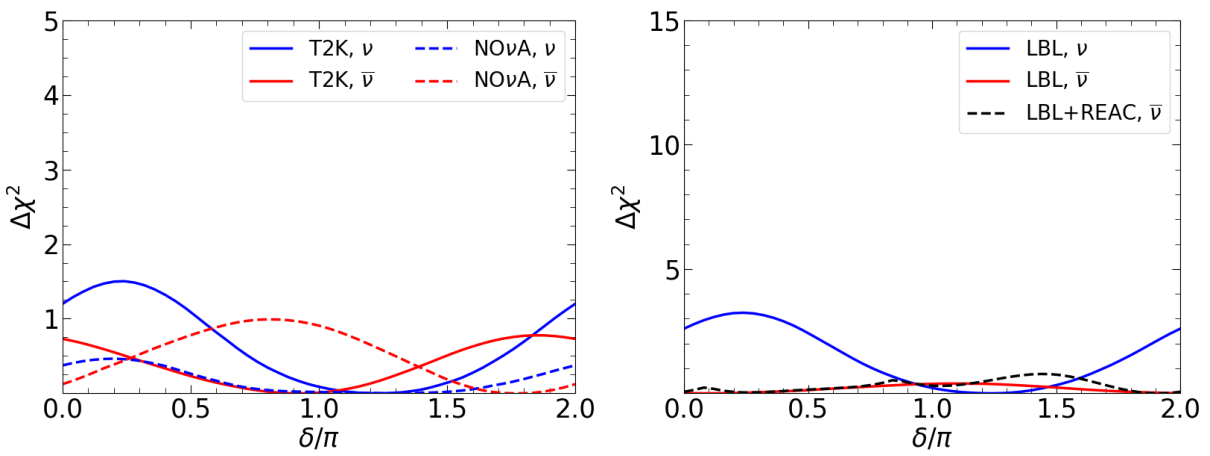

Figure 2: Left: $\Delta \chi^{2}$ profiles obtained from the analysis of neutrino (blue) and antineutrino (red) data from $\mathrm{T} 2 \mathrm{~K}$ (solid) and $\mathrm{NO} v \mathrm{~A}$ (dashed) for the $\mathrm{CP}$ phases $\delta$ (neutrinos) and $\bar{\delta}$ (antineutrinos). Right: The profiles obtained from the combination of $\mathrm{T} 2 \mathrm{~K}$ and $\mathrm{NO} v \mathrm{~A}$ data (red and blue) and from the combination of long baseline and reactor antineutrino data (black dashed).

and very close values for the best fit points obtained in both analysis. In the right panel we see that $\mathrm{NO} v \mathrm{~A}$ shows a disagreement in the best fit values of $\Delta m_{31}^{2}$ and $\sin ^{2} \theta_{23}$ obtained for neutrinos and antineutrinos. Actually, one observes that the values of $\sin ^{2} \bar{\theta}_{23}$ further away from maximal mixing are correlated to larger values of $\Delta \bar{m}_{31}^{2}$. Note that nevertheless there is significant overlap in the regions.

If we now turn to the sensitivity to the CP phases, $\delta$ and $\bar{\delta}$, we see in the left panel of Fig. 2, that all values of the CP phases remain allowed at approximately $1 \sigma$ for both experiments. This means that, unfortunately, at present, neither $\mathrm{T} 2 \mathrm{~K}$ or $\mathrm{NO} v \mathrm{~A}$ alone can make any significant statement about the measurement of the $\mathrm{CP}$ phase without assuming CPT invariance. To understand the main difference between our results and the ones obtained with the usual CPT-conserving analyses (see e.g. Ref. [5]), one should recall the origin of the sensitivity to the CP phase in those analyses. If we assume CPT invariance, for a fixed value of $\theta_{13}$ (common to neutrinos and antineutrinos), the presence of a non-zero $\mathrm{CP}$ phase can induce a shift in the neutrino oscillation probability (and therefore in the event numbers) into different directions for neutrinos and antineutrinos. If we allow, however, for the angles to be different $\left(\theta_{13} \neq \bar{\theta}_{13}\right)$, there is no need for invoking non-zero values of the CP-violating phase to reproduce the observed number of neutrino and antineutrino events, since the mixing angles can directly be adjusted to reproduce the experimental results. As a result, the sensitivity to $\mathrm{CP}$ violation in the CPT-violating scenario is very poor and, therefore, much more statistics would be necessary to disentangle the effects of $\theta_{13}$ and $\delta$ using only the neutrino or the antineutrino channel. It is then not surprising that we can not measure the $\mathrm{CP}$ phase in the separated analysis of $\mathrm{T} 2 \mathrm{~K}$ and $\mathrm{NO} v \mathrm{~A}$ data. This situation does not improve after combining the antineutrino data set with those from reactor experiments, see right panel of Fig. 2. For more details on the analyses and results we refer the reader to Ref. [6].

The data allows us to bound the CPT violating observables $|\Delta x|=|x-\bar{x}|$. The bounds on CPT violation in the neutrino sector at $3 \sigma$ are summarized in Table 1 . Note that, here we adopted the bounds on the solar parameters from Ref. [7]. 


\begin{tabular}{|c|c|c|c|c|}
\hline$\Delta\left(\Delta m_{21}^{2}\right)$ & $\Delta\left(\Delta m_{31}^{2}\right)$ & $\Delta \sin ^{2} \theta_{12}$ & $\Delta \sin ^{2} \theta_{13}$ & $\Delta \sin ^{2} \theta_{23}$ \\
\hline $4.7 \times 10^{-5} \mathrm{eV}^{2}$ & $2.5 \times 10^{-4} \mathrm{eV}^{2}$ & 0.14 & 0.029 & 0.19 \\
\hline
\end{tabular}

Table 1: Current $3 \sigma$ bounds on the differences of neutrino and antineutrino oscillation parameters.

\section{Sensitivity to CPT violation at DUNE}

To estimate the sensitivity of DUNE we assume DUNE to run 3.5 years in both neutrino and antineutrino mode. We create the fake data under the assumption of CPT conversation, using the neutrino oscillation parameters from Ref. [8], except for the atmospheric angle, for which we consider three different values, namely $\sin ^{2} \theta_{23}=0.43,0.5,0.6$. We find that the bounds on the difference of reactor angles and CP phases are not very strong [7]. However, we obtain interesting results for $\Delta\left(\Delta m_{31}^{2}\right)$ and $\Delta\left(\sin ^{2} \theta_{23}\right)$, as seen in Fig. 3. The different lines refer to the different assumed values of the atmospheric angle. Regarding mass splittings, DUNE can put stronger bounds, namely $\Delta\left(\Delta m_{31}^{2}\right)<8.1 \times 10^{-5} \mathrm{eV}^{2}$ at $3 \sigma$. For the atmospheric angle we obtain different results depending on the true value assumed in the simulation of DUNE data. The second minimum for the non-maximal values appears due to the octant degeneracy. Note that these curves have a maximum close to $\Delta \sin ^{2} \theta_{23} \approx 0.08$. A difference of this size was obtained by T2K [9] and also in Ref. [6]. We conducted a further test assuming a difference of this size as true parameters, and found that DUNE could clearly measure the difference in neutrino and antineutrino oscillations, see the left panel of Fig. 4 in comparison with the current determination in the right panel of the figure. We found that in this case DUNE could actually rule out $\Delta \sin \theta_{23}=0$ (the CPT conserving scenario) at close to $5 \sigma$ [7].
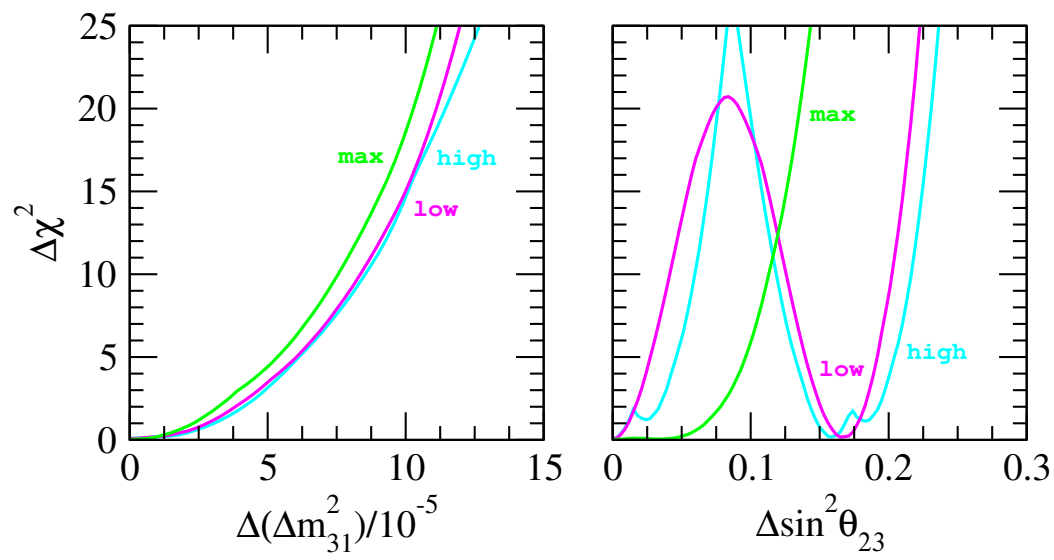

Figure 3: The sensitivity of DUNE to the difference of oscillation parameters. Here low, max and high refer to the true atmospheric angle in the lower octant, maximal and in the upper octant, respectively.

\section{Imposter solutions}

We have also shown, that if CPT is violated in nature, we can obtain fake solutions in our data analyses. This happens if CPT is violated in nature, but data are analyzed in a CPT-conserving way. Assuming for example different values of the atmospheric angles in the fake data, but analyzing the 

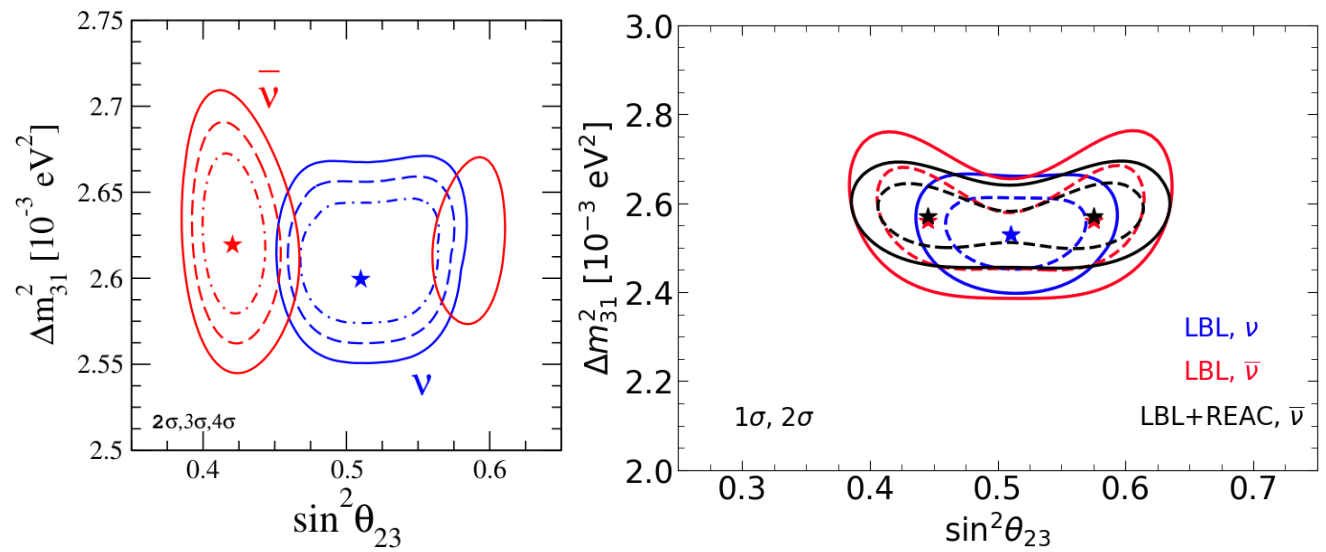

Figure 4: DUNE expected regions at $2 \sigma, 3 \sigma$ and $4 \sigma$ in the atmospheric plane (left) in comparison with the regions at $1 \sigma$ and $2 \sigma$ obtained from current data (right).

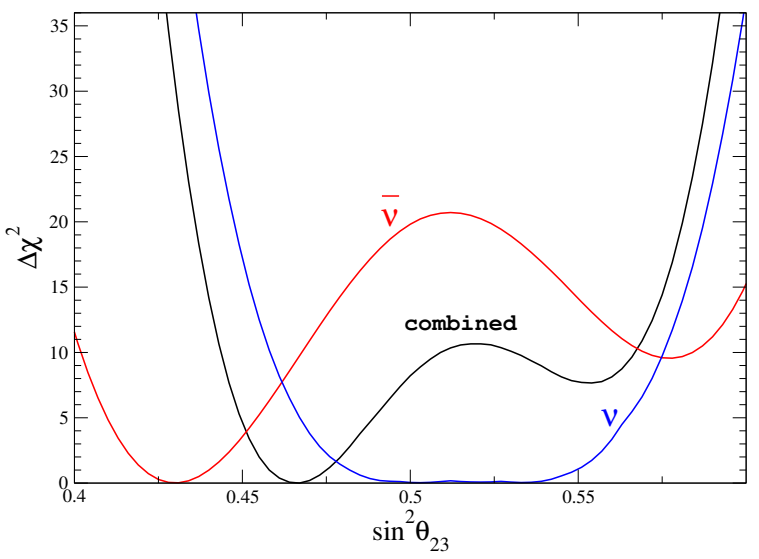

Figure 5: DUNE sensitivity to the atmospheric angle for neutrinos (blue), antineutrinos (red) and to the combination of both under the assumption of CPT conservation (black).

data in a CPT-conserving manner, we obtain a best fit value in our analysis which is different to both assumed true values as shown in Fig. 5. The blue and red lines are the individual analyses of neutrino and antineutrino data, while the black line is the result of the combined and CPT-conserving analysis. Here we see, that the combined analyzes excludes the true values at close to $3 \sigma$ in one case and more than $5 \sigma$ in the other. In Ref. [7] we have shown that a similar problem can arise in the determination of $\delta$.

\section{Mimicking effects between CPT violation and non-standard interactions}

Matter effects can induce an intrinsic CPT violation through neutrino non-standard interactions (NSI). We use the same data that we generated for the creation of Fig. 4 (left panel), and analyze it in a CPT-conserving way but this time we include the NSI parameters $\epsilon_{\mu \tau}^{m}$ and $\epsilon_{\tau \tau}^{m}$ in our analysis (see Refs. [10,11] for the details of NSI). We find that the data can be fit very well with a non-zero $\epsilon_{\tau \tau}^{m}$. The profiles for the NSI parameters obtained from our analysis in comparison with bounds taken for Ref. [10] are shown in Fig. 6. Therefore, one can conclude that what seems to be CPT 


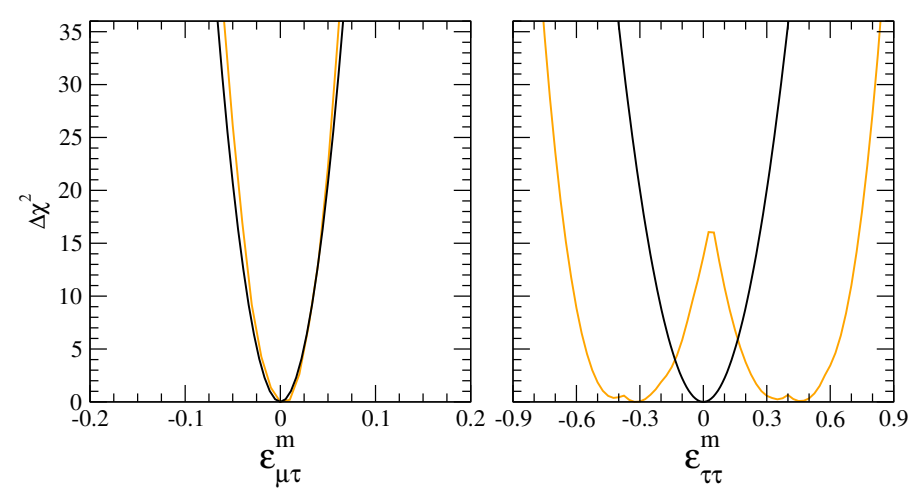

Figure 6: $\Delta \chi^{2}$ profiles for the NSI couplings $\epsilon_{\mu \tau}^{m}$ (left) and $\epsilon_{\tau \tau}^{m}$ (right panel) obtained in our analysis (orange lines). For comparison we show the experimental bounds on both parameters assuming Gaussian errors (black lines), taken from Ref. [10].

violation could be also explained with NSI. Even though the best fit value seems to be excluded by current data, there could be a "transition zone" where both CPT violation and NSI would give reasonably good fits of the data.

\section{Acknowledgments}

Work supported by the research grant "The Dark Universe: A Synergic Multimessenger Approach" number 2017X7X85K under the program "PRIN 2017" funded by the Ministero dell'Istruzione, Università e della Ricerca (MIUR).

\section{References}

[1] T2K collaboration, Constraint on the matter-antimatter symmetry-violating phase in neutrino oscillations, Nature 580 (2020) 339 [1910. 03887].

[2] NOvA collaboration, First Measurement of Neutrino Oscillation Parameters using Neutrinos and Antineutrinos by NOvA, Phys. Rev. Lett. 123 (2019) 151803 [1906. 04907].

[3] Daya BAy collaboration, Measurement of the Electron Antineutrino Oscillation with 1958 Days of Operation at Daya Bay, Phys. Rev. Lett. 121 (2018) 241805 [1809. 02261].

[4] RENO collaboration, Measurement of Reactor Antineutrino Oscillation Amplitude and Frequency at RENO, Phys. Rev. Lett. 121 (2018) 201801 [1806. 00248].

[5] P. F. de Salas, D. V. Forero, S. Gariazzo, P. Martínez-Miravé, O. Mena, C. A. Ternes et al., 2020 global reassessment of the neutrino oscillation picture, JHEP 02 (2021) 071 [2006.11237].

[6] M. A. Tórtola, G. Barenboim and C. A. Ternes, CPT and CP, an entangled couple, JHEP 07 (2020) 155 [2005.05975]. 
[7] G. Barenboim, C. A. Ternes and M. Tórtola, Neutrinos, DUNE and the world best bound on CPT invariance, Phys. Lett. B 780 (2018) 631 [1712.01714].

[8] P. F. de Salas, D. V. Forero, C. A. Ternes, M. Tortola and J. W. F. Valle, Status of neutrino oscillations 2018: $3 \sigma$ hint for normal mass ordering and improved CP sensitivity, Phys. Lett. B 782 (2018) 633 [1708.01186].

[9] T2K collaboration, Updated T2K measurements of muon neutrino and antineutrino disappearance using $1.5 \times 10^{21}$ protons on target, Phys. Rev. D96 (2017) 011102 [1704.06409].

[10] Y. Farzan and M. Tortola, Neutrino oscillations and Non-Standard Interactions, Front. in Phys. 6 (2018) 10 [1710.09360].

[11] G. Barenboim, C. A. Ternes and M. Tórtola, New physics vs new paradigms: distinguishing CPT violation from NSI, Eur. Phys. J. C 79 (2019) 390 [1804 . 05842]. 